\title{
Development of a magnetic resonance imaging protocol to visualize encapsulated contrast agent markers in prostate brachytherapy recipients: initial patient experience
}

\author{
Tze Yee Lim, PhD',2, Rajat J. Kudchadker, PhD², Jihong Wang, PhD², Tharakeswara Bathala, MD³, Janio Szklaruk, MD4, \\ Thomas J. Pugh, MD5 , Usama Mahmood, MD ${ }^{5}$, Geoffrey S. Ibbott, PhD², Steven J. Frank, MD ${ }^{5}$ \\ University of Texas at Houston Graduate School of Biomedical Sciences, Houston, TX, ${ }^{2}$ Department of Radiation Physics, University of Texas \\ MD Anderson Cancer Center, Houston, TX, ${ }^{3}$ Department of Diagnostic Radiology, University of Texas MD Anderson Cancer Center, Houston, TX, \\ ${ }^{4}$ Department of Diagnostic Radiology, University of Texas MD Anderson Cancer Center, Houston, TX, ${ }^{5}$ Department of Radiation Oncology. \\ University of Texas MD Anderson Cancer Center, Houston, TX, USA
}

\begin{abstract}
Purpose: Computed tomography (CT)-based prostate post-implant dosimetry allows for definitive seed localization but is associated with high interobserver variation in prostate contouring. Currently, magnetic resonance imaging (MRI)-based post-implant dosimetry allows for accurate anatomical delineation but is limited due to inconsistent seed localization. Encapsulated contrast agent markers were previously proposed to overcome the seed localization limitation on MRI images by placing hyperintense markers adjacent to hypointense seeds. The aim of this study was to assess the appearance of these markers in prostatic tissue, and develop an MRI protocol to enable marker visualization.

Material and methods: We acquired MRI scans in prostate implant patients $(n=10)$ on day 0 (day of implant) and day 30 (month after implant). Before implantation of the markers, the routine post-implant MRI protocol included a 3D T2-weighted fast-spin-echo (FSE) sequence with which markers and seeds could not be clearly visualized. To visualize the MRI markers, a 3D fast radiofrequency-spoiled gradient-recalled echo (FSPGR) sequence was evaluated for marker and seed visibility, as well as prostate boundary definitions.

Results: The 3D FSPGR sequence allowed for the visualization of markers in the prostate, enabling the distinction of signal voids as seeds versus needle tracks. The updated post-implant MRI protocol consists of this 3D FSPGR scan and an optional 3D T2-weighted FSE scan. The optional 3D T2-weighted FSE sequence may be employed to better visualize intraprostatic detail. We also described the observed image artifacts, including seed susceptibility, marker chemical shift, partial volume averaging, motion, and wraparound artifacts.

Conclusions: We have demonstrated an MRI protocol for use with hyperintense encapsulated contrast agent markers to assist in the identification of hypointense seeds.
\end{abstract}

Key words: brachytherapy, dosimetry markers, MRI, prostate cancer, seeds.

\section{Purpose}

Post-implant quality assessment is important to ensure that the radiation dose adequately covers the prostate and is acceptable in nearby critical structures. Implant quality can be represented by dose-volume histogram (DVH) parameters, which correlate with biochemical control and survival $[1,2,3,4]$. For the calculation of DVH parameters, two vital pieces of information are needed: dose information from the seed locations, and volume information using the boundaries of the prostate and surrounding critical structures. Seed localization and anatomical delineation are dependent on the post-implant imaging modality.

Computed tomography (CT) is the current standard post-implant imaging modality, as the metallic radioactive seeds' high atomic number and density result in high contrast between the seeds and prostatic tissue. However, the prostate is not well demarcated from surrounding tissues with similar densities, and combined with the seed streak artifacts that obscure prostate boundaries, prostate vol- 
umes can be overestimated on CT images $[5,6,7]$. High interobserver variation in prostate volume delineation on $\mathrm{CT}$ images $[7,8]$ results in increased variability in post-implant dosimetry and obscures dose-response relationships [9].

Magnetic resonance imaging (MRI) is an alternate post-implant imaging modality. Currently, MRI is the optimum imaging modality for staging of a suspected primary prostate malignancy. The primary advantage of MRI compared to CT is that a variety of MRI techniques allows for better anatomical visualization, such as intraprostatic detail, the prostate apex and base, internal pudendal artery, neurovascular bundles, penile bulb, bladder neck and urinary sphincters $[6,7,10,11,12,13]$. The low interobserver variation in prostate contouring on MRI scans $[7,8]$ would allow consistent reporting of dosimetric outcomes. Consistent and accurate quantitation of dose to critical structures, such as the urinary sphincter [13], would improve evaluation of dose-response relationships. The main drawback of using MRI for post-implant dosimetry is that the seeds generally appear as hypointense signal voids that may be mistaken for needle tracks or blood vessels $[14,15]$.

MRI-CT fusion post-implant dosimetry allows for anatomical definition on MRI images and seed localization on CT images. Nonetheless, the MRI-CT fusion quality is affected by registration quality (registration landmarks, fusion technique, fusion algorithm parameters, personnel training and experience) and imaging quality (pulse sequence, contrast, pelvic tilt, coil, patient transit between scanners, duration between scans, bladder and rectal filling differences) $[16,17,18,19]$.

The advantages of MRI-only post-implant dosimetry are superior soft tissue contrast, no extraneous radiation, imaging flexibility, and possible integration of functional imaging. However, MRI is not as widely adopted as CT in prostate brachytherapy clinics owing to seed localiza- tion difficulties, image fusion issues, and logistical barriers. To assist in definitive seed localization on MRI scans, Frank et al. [10] developed encapsulated contrast agent markers that can be placed in between seeds (Figure 1). The markers do not affect dose distribution [20,21], have minimal toxicity [22], and have well-defined MRI relaxation properties $[23,24]$.

In this manuscript, we describe our experience in developing an appropriate MRI protocol for marker visualization, and the process of incorporating the markers into our low dose rate (LDR) prostate brachytherapy clinical practice.

\section{Material and methods}

In an institutional process quality improvement protocol, 10 prostate cancer patients selected to undergo LDR prostate brachytherapy were evaluated. Various imaging modalities, such as transrectal ultrasound (TRUS), $\mathrm{CT}$, and MRI were used throughout the implant workflow. Prior to the implant, TRUS and MRI images were acquired. These pre-implant images were used to generate the treatment plan on MIM Symphony (Cleveland, $\mathrm{OH}, \mathrm{USA})$, which has been previously commissioned [25]. The treatment plan dictated the ordering of seeds and markers. The markers (Sirius ${ }^{\mathrm{TM}}$; C4 Imaging, Houston, TX, USA) contain cobalt dichloride-N-acetyl cysteine encapsulated in a polymer capsule of $5.5 \mathrm{~mm}$ length, $0.8 \mathrm{~mm}$ diameter, and have been approved by the United States Food and Drug Administration for LDR prostate implants. The seed-marker strands were ordered directly from the seed manufacturer depending on the isotope (IsoRay Medical, Richland, WA, USA; IsoAid, Port Richey, FL, USA; Theragenics, Buford, GA, USA).

The seed-marker strands were implanted under TRUS guidance. After the implant, CT and MRI scans were ac-
A

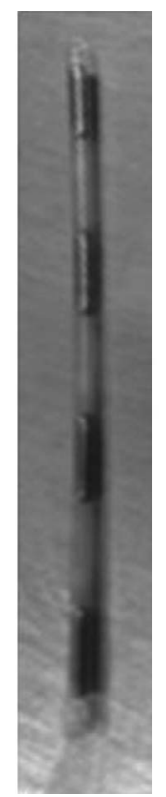

B

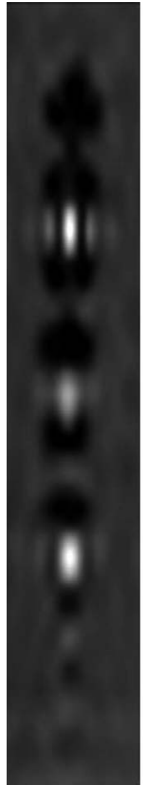

C

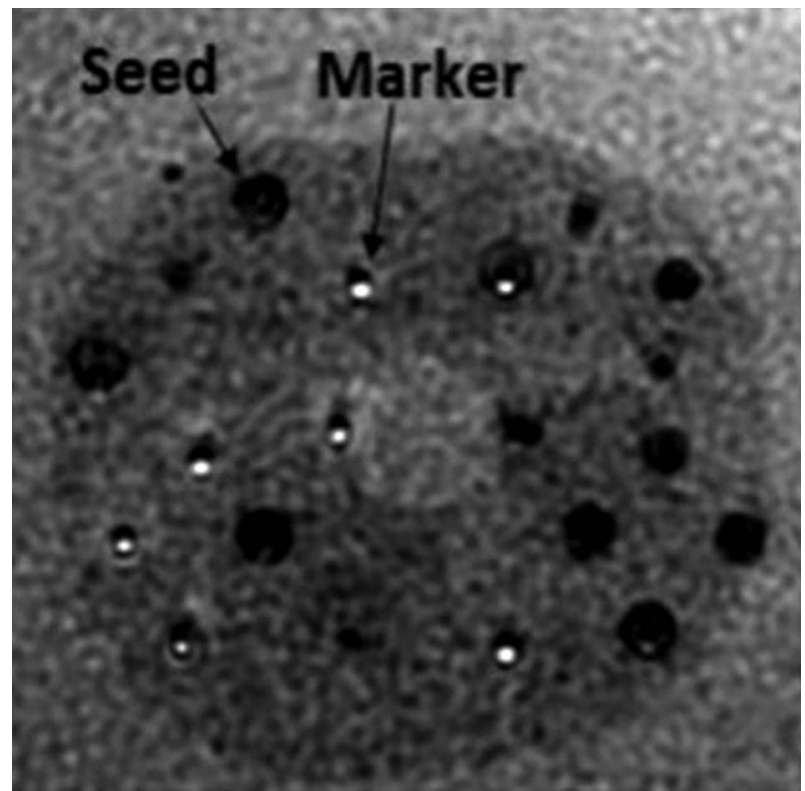

Fig. 1. A) Photograph of markers between brachytherapy seeds. B) The appearance of markers (hyperintense cylinders) between seeds (hypointense dumbbell-shaped voids) in a commercially-available prostate phantom. The displayed sagittal slice was obtained at a plane crossing the center of the topmost marker. C) Axial view in a prostate phantom. Markers appear definitively as hyperintense regions, whereas signal voids may be seeds or needle tracks 
quired on the day of implant and again approximately a month later. Post-implant CT images were used to perform post-implant dosimetry for all patients according to the current standard of care. Post-implant MRI images were evaluated for marker, seed and prostatic anatomy visibility, as well as artifacts, including marker chemical shift, partial volume averaging, seed susceptibility, motion, and wraparound artifacts.

The previous post-implant MRI protocol consisted of three dimensional (3D) $\mathrm{T}_{2}$-weighted fast spin echo (FSE), two dimensional (2D) $\mathrm{T}_{2}$-weighted FSE (axial), and $2 \mathrm{D} \mathrm{T}_{1}$-weighted FSE (axial/sagittal/coronal) sequences. The 3D $T_{2}$-weighted FSE sequence (GE: CUBE; Siemens: SPACE, Sampling Perfection with Application optimized Contrasts using different flip angle Evolution) was routinely used for fusion with CT images.

The updated post-implant MRI protocol consists of a $3 \mathrm{D}$ fast radiofrequency-spoiled gradient-recalled echo (FSPGR) sequence, with the scan parameters defined based on previous phantom studies [24] and repeat 3D FSPGR scans performed to optimize the sequence for post-implant dosimetry (Table 1). MRI scans were acquired using a Signa HDxt 3.0T scanner (GE, Waukesha, WI, USA) for eight patients, a Signa HDxt $1.5 \mathrm{~T}$ scanner (GE, Waukesha, WI, USA) for one patient, and a MAGNETOM Aera 1.5 T scanner (Siemens, Malvern, PA, USA) with the similar fast low angle shot (FLASH) sequence for one patient. Except for the first patient, all patients were imaged with a disposable inflatable endorectal coil (Medrad ${ }^{\circledR}$; Bayer, Whippany, NJ, USA). Surface coil intensity correction (SCIC) and phased array uniformity enhancement (PURE) post-processing were applied to minimize the high signal intensity proximal to the endorectal coil. All patients were imaged in the supine position.

\section{Results}

On the CT images (Figure 2), the markers appeared isointense to the prostate tissue and were obscured by the seeds. On the other hand, the seeds appeared as hyperintense cylinders. The seed positions were confounded by the metal streak artifacts and partial volume averaging artifacts, appearing longer and wider than the seeds' physical dimensions. On the standard post-implant protocol's 3D T $\mathrm{T}_{2}$-weighted FSE images (Figure 3), the markers and seeds appeared inconsistently as hypointense cylinders or isointense to prostatic tissue. Unclear distinction between seeds and markers confounded seed localization. Prostate anatomy was better visualized on the 3D $\mathrm{T}_{2}$-weighted FSE images compared to CT images.

On the updated protocol's 3D FSPGR images (Figure 4), the markers appeared as hyperintense cylinders within the hypointense needles tracks, giving the characteristic appearance on axial images of bright filled circles with a dark outline. A chemical shift artifact of the marker was pronounced at low bandwidth (BW). The marker chemical shift artifact results from slight differences between the Larmor frequencies of hydrogen spins in the marker and the prostate, thereby displacing the marker in the frequency encoding direction. The presence and magnitude of this displacement depends on BW and matrix size. The BW should be high enough for minimal marker displacement but low enough for acceptable noise. To reduce partial volume averaging artifacts due to the small size of the markers, we used $0.27 \times 0.27 \times 1 \mathrm{~mm}^{3}$ voxels. The number of averages used was relatively high compared to standard sequences to increase the signal-tonoise ratio (SNR).

On the other hand, the seeds appeared as hypointense dumbbell shapes, with wider widths at the

Table 1. Pulse sequence parameters for post-implant dosimetric assessment

3D T2W FSE

\begin{tabular}{|c|c|c|c|c|}
\hline Scanner manufacturer & GE & GE & GE & Siemens \\
\hline Field strength $(\mathrm{T})$ & 3.0 & 3.0 & 1.5 & 1.5 \\
\hline Scan plane & Axial & Axial & Axial & Axial \\
\hline Repetition time/Echo time (ms) & $2000 / 120$ & $8 / 2$ & $8 / 3.6$ & $12 / 2.4$ \\
\hline Field-of-view ${ }^{a}(\mathrm{~cm})$ & 14 & 14 & 16 & 14 \\
\hline Bandwidth (Hz/pixel) & 122 & 326 & 244 & 500 \\
\hline Echo train length & 74 & - & - & - \\
\hline Flip angle $\left(^{\circ}\right)$ & - & 20 & 25 & 25 \\
\hline Slice thickness $(\mathrm{mm})$ & $1^{b}$ & $1^{b}$ & $1^{b}$ & 1 \\
\hline Matrix size & $512 \times 512$ & $512 \times 512$ & $512 \times 512$ & $512 \times 512$ \\
\hline Number of averages & 1 & $8^{\mathrm{d}}$ & $9^{d}$ & 2 \\
\hline Frequency direction & $R / L$ & $A / P$ & $A / P$ & $A / P$ \\
\hline
\end{tabular}

asquare field-of-view

Interpolated from $2 \mathrm{~mm}$

Interpolated from $256 \times 256$

'Halved due to phase oversampling 


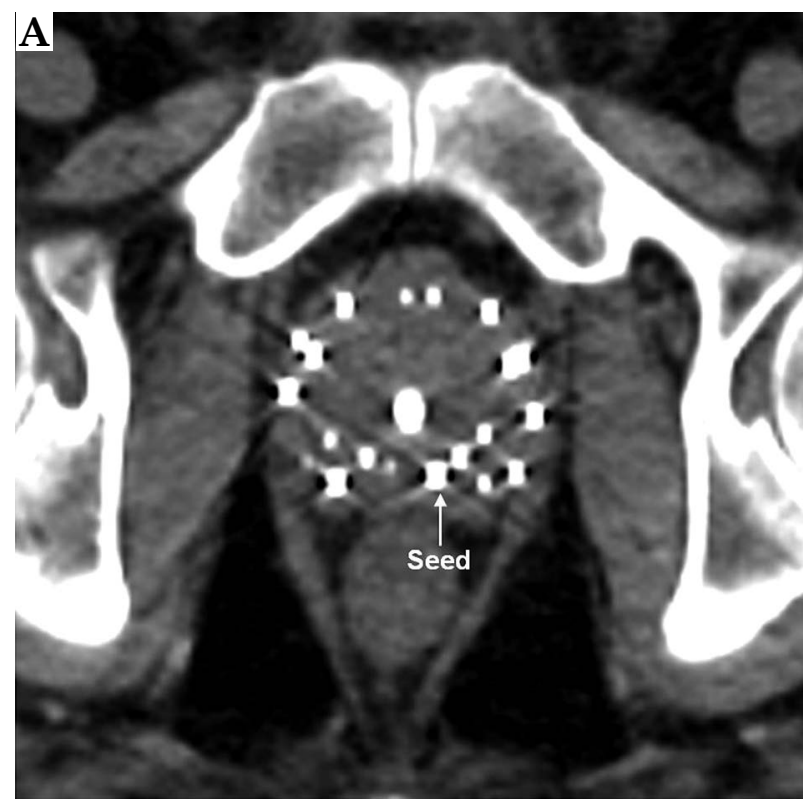

Fig. 2. Axial (A), sagittal (B), and coronal (C) views of the prostate acquired using CT. Markers could not be visualized. Seeds appeared hyperintense with streak artifacts

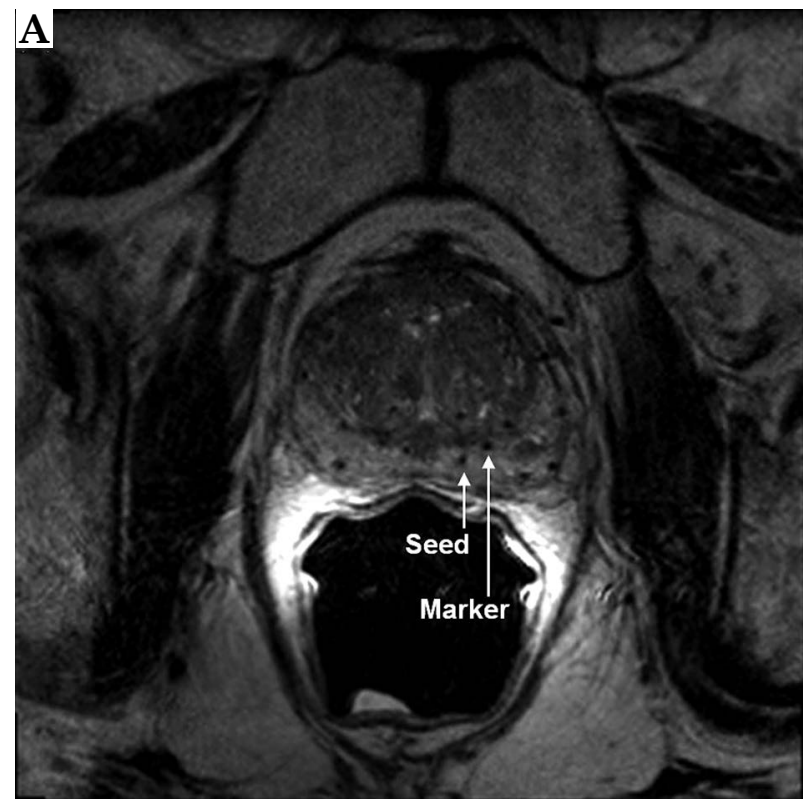

Fig. 3. Axial (A), sagittal (B), and coronal (C) views of the prostate acquired using a 3D fast spin echo sequence. The hypointense seeds and markers were not clearly distinguishable

ends of the seeds compared to the center of the seeds. These dumbbell shaped seed susceptibility artifacts were seen as the magnetic field inhomogeneity was distorted owing to the higher magnetic permeability of the seeds' metallic casing, especially at the end-welding,
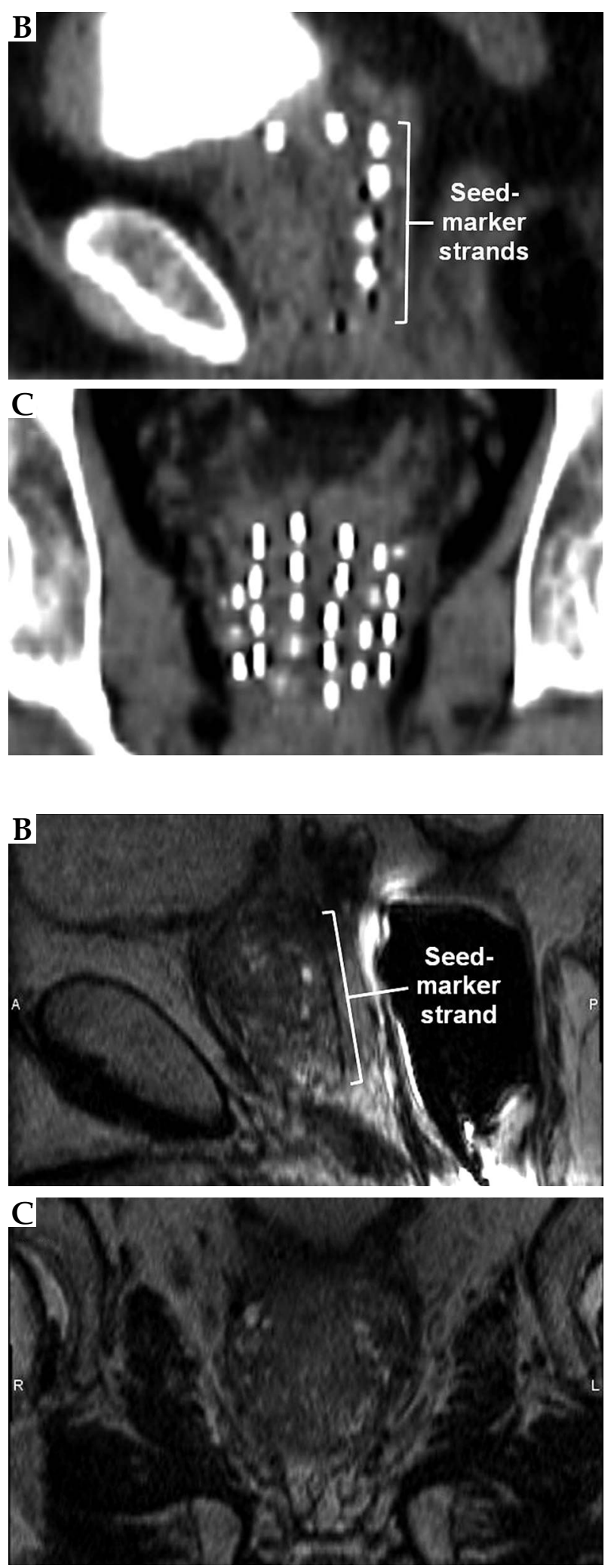

compared to background prostatic tissue. The seed susceptibility artifact was more pronounced at low BW and more visible on 3D FSPGR images compared to 3D FSE images due to the lack of a $180^{\circ}$ pulse that cancels out magnetic field inhomogeneities. The seed susceptibility 


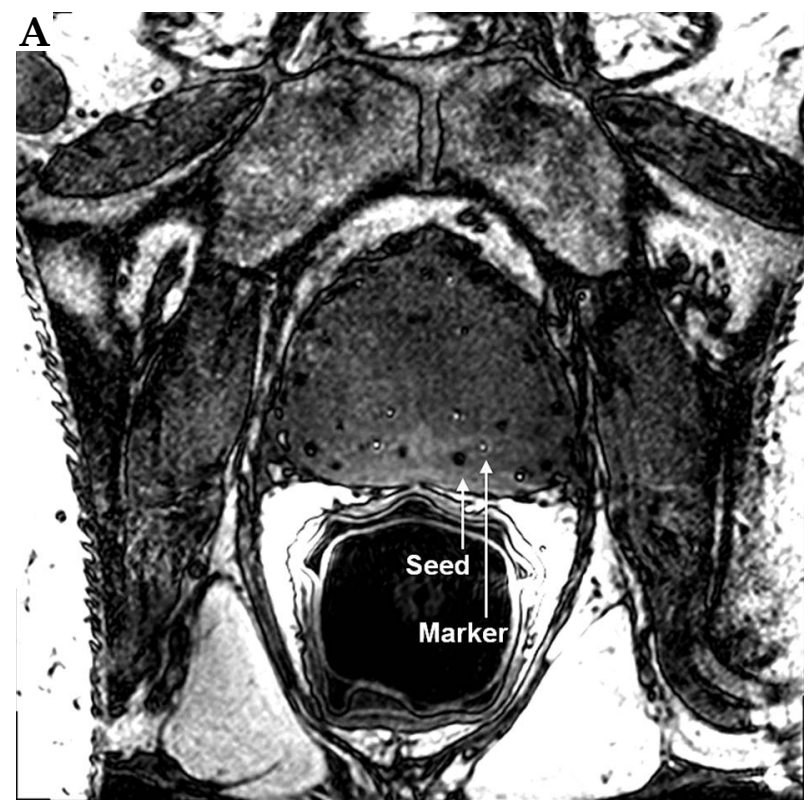

Fig. 4. Axial (A), sagittal (B), and coronal (C) views of the prostate acquired using a 3D fast spoiled gradient echo sequence. Markers appeared as hyperintense cylinders, while seeds appeared as hypointense dumbbell-shaped susceptibility voids

artifact, along with the markers, enabled easier seed centroid identification.

Intraprostatic detail was not as clearly visualized with the 3D FSPGR sequence (Figure 4) compared to the 3D $\mathrm{T}_{2}$-weighted FSE sequence (Figure 3). Nevertheless, for post-implant dosimetry purposes, only prostate boundary delineation is needed.

Patient motion caused a ripple appearance in the phase encoding direction due to improper registration
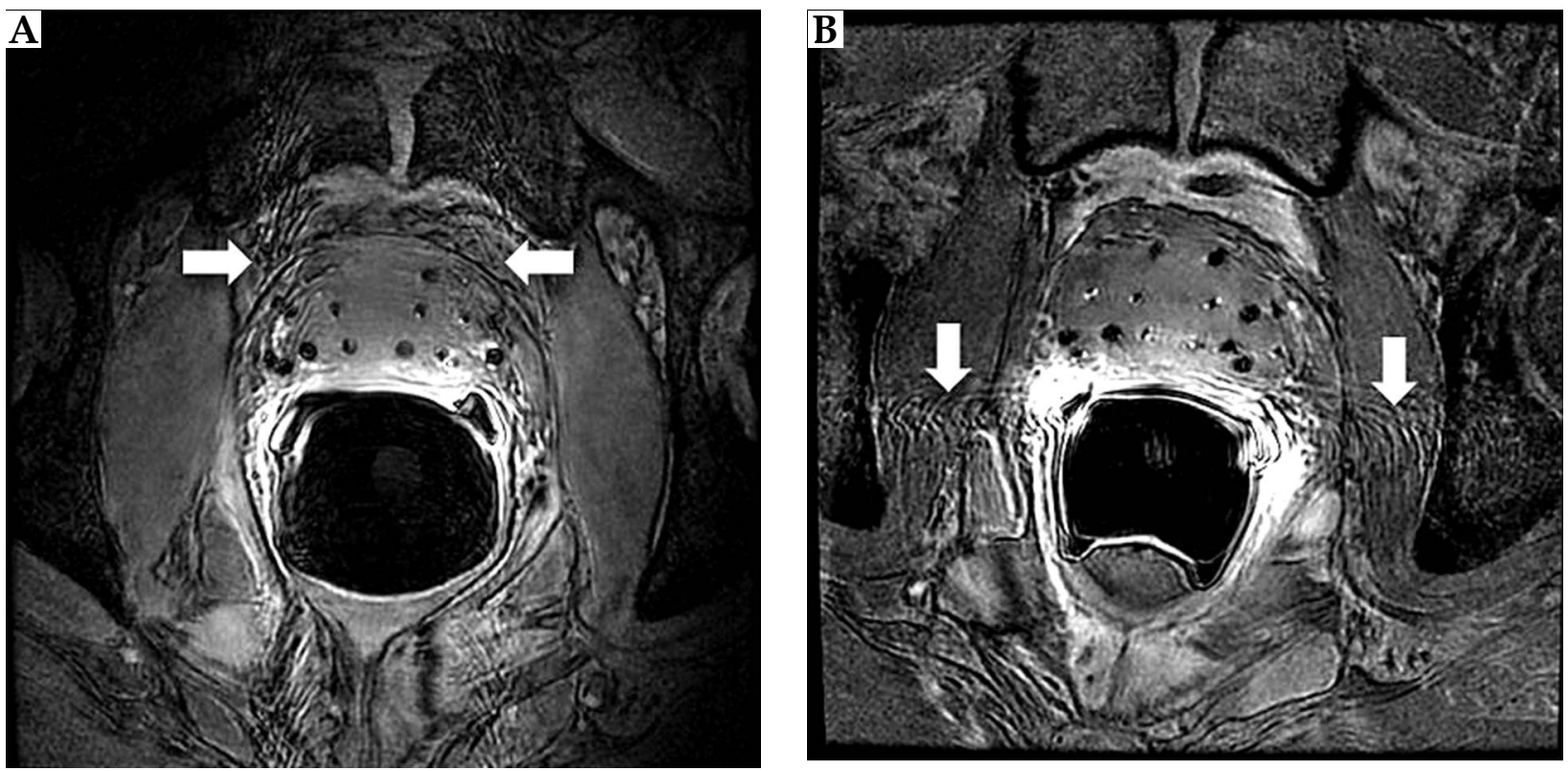

Fig. 5. Motion artifact on axial views of the prostate acquired using a 3D FSPGR sequence. A) Phase-encoding direction = anterior $/$ posterior. B) Phase-encoding direction $=$ right $/$ left 

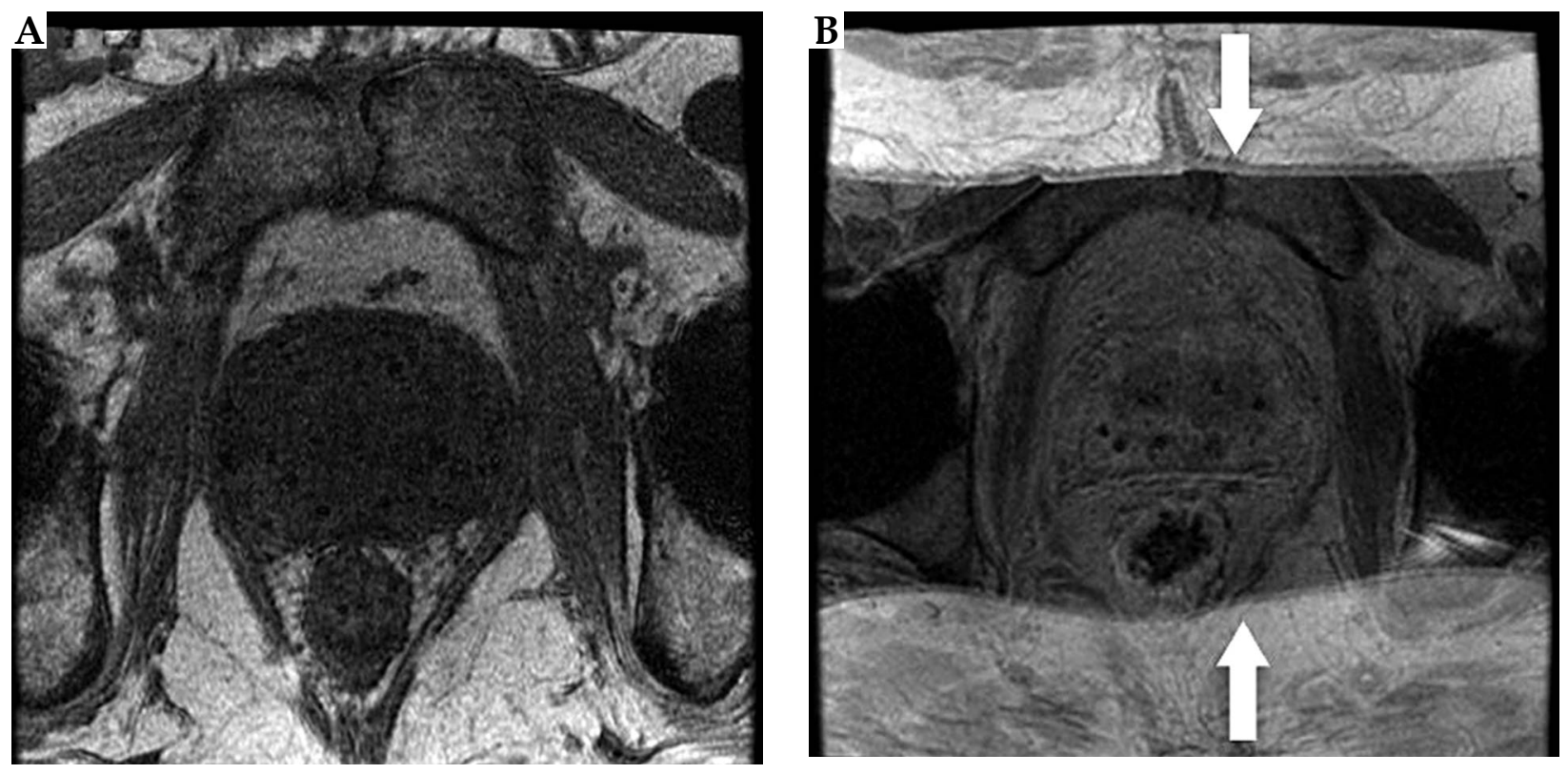

Fig. 6. Axial views of the prostate acquired using a 3D FSPGR sequence with no wrap artifact (A) versus with wrap artifact (B)

of phase shift information and could affect marker visibility. To reduce this artifact, a common technique is to set the phase encoding direction to anterior/posterior because the anteroposterior width usually has the shortest skin-to-skin distance. However, since the imaging FOV was smaller than the anteroposterior width of most patients, patient anatomy outside the FOV would wrap into the image (Figure 6). To reduce the wraparound artifact, we oversampled the FOV, used saturation bands to reduce superimposed signal from outside the imaging FOV, and kept the patient's arms away from their sides.

Minimizing the motion artifact and wraparound artifact required the phase-encoding to be set in different directions. Nevertheless, using phase oversampling, the wraparound artifact would not extend into the prostate; hence preventing the motion artifact from impinging into the prostate region could be prioritized to ensure visualization of the prostate boundaries and markers/seeds within the prostate for the purposes of post-implant dosimetric evaluation. Therefore, the phase-encoding direction should be set to R/L. For patients with lateral widths greater than the oversampled FOV, the wrap artifact would be observable (hyperintense tissue visible on $\mathrm{R} / \mathrm{L}$ edges of the axial view Figure $4 \mathrm{~A}$ and coronal view Figure 4C).

Patients' biomedical implants may be contraindicated for 3.0 T MRI but may be allowed to be scanned at lower field strengths. A reduction in field strength corresponds to a reduction in power deposition in the patient, relaxation times, susceptibility artifact effect, SNR, and geometric distortion. At $1.5 \mathrm{~T}$, the image quality was reduced compared to $3.0 \mathrm{~T}$ images but the visibility of the markers was not compromised.

Endorectal coils are routinely used to enhance the prostate signal for diagnostic sequences, as the prostate is centered in the body while signal from the torso coil falls off away from the skin. Identification of the seeds and markers was difficult without the endorectal coil (Figure 6A). However, endorectal coils may deform the prostate, resulting in MRI-CT fusion difficulties and producing unnatural dose distributions. To reduce prostate deformation while maintaining coil immobility, the endorectal coil was slightly inflated to only $30 \mathrm{cc}$ instead of maximum inflation.

\section{Discussion}

Post-implant dosimetry improves care by allowing for corrective measures to be taken if necessary, and can improve the care of future patients through implant quality feedback to the brachytherapy team. Communication of the methodology and end-points of post-implant dosimetric assessment by the brachytherapy team to the MRI team is crucial to ensure useful images are acquired while maintaining high SNR, minimal artifacts, and reasonable scan time.

In this study, we described our experience in developing an MRI protocol for marker and prostate visualization, and the incorporation of markers into our LDR prostate brachytherapy clinical practice. This is the first study presenting the appearance of MRI markers in human prostate, and the first evaluation of the practical feasibility of using these markers as part of the LDR prostate brachytherapy workflow. Our MRI protocol consists of a 3D FSPGR scan for marker visualization and an optional 3D $\mathrm{T}_{2}$-weighted FSE scan for detailed anatomical visualization. The 3D FSPGR scan may be used as the sole image set to identify markers and seeds, and provides adequate prostate edge visualization for contouring. However, MRI-CT fusion can be done using either the 3D FSPGR or 3D FSE scans in our protocol for fusion with CT images, if desired. Especially on the 3D FSPGR images, the markers can be visualized, potentially allowing for greater registration to CT images, as the markers and seeds are interleaved. MRI-MRI fusion post-implant dosimetry can also be straightforwardly done with our protocol's 3D FSPGR and 3D FSE scans, as these scans were 
acquired consecutively using an endorectal coil with the same scan prescription.

A limitation of the study is that the effects of different scan parameters, scan conditions, and scanner manufacturers could not be comprehensively studied due to scanner time constraints, patient comfort concerns, and the small patient sample size. On the other hand, based on the imaging findings of this study, we are currently acquiring MRI images of more patients implanted with markers to investigate if there is any clinically significant difference of DVH parameters between CT-only and MRI-only post-implant dosimetry.

Regardless of the MRI scanner manufacturer, consistent and uniform protocols are essential. The MRI markers could be visualized on both GE and Siemens scanner platforms. The convenience of using the clinically-available spoiled gradient echo pulse sequence compared to novel pulse sequences for MRI-based post-implant dosimetry was that the scan parameters could be matched with similar pulse sequences of different scanner manufacturers

The advantages of using markers for MRI-based post-implant dosimetry are easier identification of hyperintense markers compared to hypointense seeds, prevention of spacers being incorrectly identified as seeds, and distinction between needle tracks and blood vessels. Conversely, the limitations of MRI-based post-implant dosimetry with markers include the indirect correspondence between hyperintense signals and seed positions, restriction to stranded use, difficult localization of migrated seeds, and manual marker-seed finding. However, compared to loose seeds, stranded seeds rarely migrate $[26,27]$.

Future work includes a semi-automated marker-seed finding algorithm to facilitate better integration of the markers into busy brachytherapy clinics. Additionally, given the feasibility of incorporating markers into the LDR prostate brachytherapy clinic as presented in this study, more investigations are needed to evaluate the efficacy of marker-based MRI-only post-implant dosimetry. The precision of DVH parameters generated from CT images, MRI-CT fusion images, and MRI images with the use of the markers could be compared. More importantly, the efficacy of these DVH parameters for the prediction of tumor control as well as acute and late effects of LDR prostate brachytherapy could be studied. Furthermore, rigid endorectal coils with a smaller diameter compared to inflatable endorectal coils may be investigated to reduce prostate deformation while maintaining marker conspicuity. Another potential future investigation is the incorporation of functional imaging sequences into the MRI protocol for early prediction of treatment response.

\section{Conclusions}

Barriers to the adoption of MRI for post-implant dosimetry are seed localization, time, cost, imaging complexity, and personnel education. We have illustrated an MRI protocol for visualization of markers that may help overcome the barrier of seed localization and spur the use of MRI for post-implant dosimetry. MRI-based post-implant dosimetry may reduce inconsistencies in target de- lineation and subsequent reporting of DVH parameters. With more accurate DVH parameters, the dose-response relationship for critical organs and subsequent toxicity can be studied in a more meaningful manner, such that we can better manage the dose delivered to the prostate and surrounding critical structures in the future.

\section{Disclosure}

Steven J. Frank is a cofounder of C4 Imaging, LLC, manufacturer of the MRI markers.

The other authors report no conflict of interest.

\section{References}

1. Stock RG, Stone NN, Tabert A et al. A dose-response study for I-125 prostate implants. Int J Radiat Oncol Biol Phys 1998; 41: 101-108.

2. Potters L, Morgenstern C, Calugaru E et al. 12-year outcomes following permanent prostate brachytherapy in patients with clinically localized prostate cancer. J Urol 2005; 173: 1562-1566.

3. Ash D, Al-Qaisieh B, Bottomley D et al. The correlation between D90 and outcome for I-125 seed implant monotherapy for localized prostate cancer. Radiother Oncol 2006; 79: 185-189.

4. Stone NN, Potters L, Davis BJ et al. Multicenter analysis of effect of high biologic effective dose on biochemical failure and survival outcomes in patients with Gleason score 7-10 prostate cancer treated with permanent prostate brachytherapy. Int J Radiat Oncol Biol Phys 2009; 73: 341-346.

5. Rasch C, Barillot I, Remeijer P et al. Definition of the prostate in CT and MRI: a multi-observer study. Int I Radiat Oncol Biol Phys 1999; 43: 57-66.

6. McLaughlin PW, Narayana V, Drake DG et al. Comparison of MRI pulse sequences in defining prostate volume after permanent implantation. Int I Radiat Oncol Biol Phys 2002; 54: 703-711.

7. Smith WL, Lewis C, Bauman G et al. Prostate volume contouring: a 3D analysis of segmentation using 3DTRUS, CT and MR. Int J Radiat Oncol Biol Phys 2007; 67: 1238-1247.

8. Dubois DF, Prestidge BR, Hotchkiss LA et al. Intraobserver and interobserver variability of MR imaging- and CT-derived prostate volumes after transperineal interstitial permanent prostate brachytherapy. Radiology 1998; 207: 785-789.

9. Crook J, Milosevic M, Catton P et al. Interobserver variation in post-implant computed tomography contouring affects quality assessment of prostate brachytherapy. Brachytherapy 2002; 1: 66-73.

10. Frank SJ, Stafford RJ, Bankson JA et al. A novel MRI marker for prostate brachytherapy. Int J Radiat Biol Phys 2008; 71: 5-8.

11. Gillan C, Kirilova A, Landon A et al. Radiation dose to the internal pudendal arteries from permanent seed prostate brachytherapy as determined by time-of-flight MR angiography. Int J Radiat Oncol Biol Phys 2006; 65: 688-693.

12. Wright JL, Newhouse JH, Laguna JL et al. Localization of neurovascular bundles on pelvic $\mathrm{CT}$ and evaluation of radiation dose to structures putatively involved in erectile dysfunction after prostate brachytherapy. Int J Radiat Oncol Biol Phys 2004; 59: 426-435.

13. Register SP, Kudchadker RJ, Levy LB et al. An MRI-based dose-response analysis of urinary sphincter dose and urinary morbidity after brachytherapy for prostate cancer in a phase II prospective trial. Brachytherapy 2013; 12: 210-216.

14. Moerland MA, Wijrdeman HK, Beersma R et al. Evaluation of permanent I-125 prostate implants using radiography and magnetic resonance imaging. Int J Radiat Oncol Biol Phys 1997; 37: 927-933. 
15. Dubois DF, Prestidge BR, Hotchkiss LA et al. Source localization following permanent transperineal prostate interstitial brachytherapy using magnetic resonance imaging. Int J Radiat Oncol Biol Phys 1997; 39: 1037-1041.

16. Roberson PL, McLaughlin PW, Narayana V et al. Use and uncertainties of mutual information for computed tomography/magnetic resonance (CT/MR) registration post permanent implant of the prostate. Med Phys 2005; 32: 473-482.

17. Maletz KL, Ennis RD, Ostenson J et al. Comparison of CT and MR-CT fusion for prostate post-implant dosimetry. Int J Radiat Oncol Biol Phys 2012; 82: 1912-1917.

18. Bowes D, Crook JM, Rajapaskshe R et al. Defining a magnetic resonance scan sequence for permanent seed prostate brachytherapy post-implant assessment. Brachytherapy 2013; 12: 25-29.

19. Kunogi H, Hojo H, Wakumoto $Y$ et al. A new two-step accurate CT-MRI fusion technique for post-implant prostate cancer. J Contemp Brachytherapy 2015; 7: 117-121.

20. Frank SJ, Tailor RC, Kudchadker RJ et al. Anisotrophy characterization of I-125 seed with attached encapsulated cobalt chloride complex contrast agent markers for MRI based prostate brachytherapy. Med Dosim 2011; 36: 200-205.

21. Melhus CS, Mikell JK, Frank SJ et al. Dosimetric influence of seed spacers and end-weld thickness for permanent prostate brachytherapy. Brachytherapy 2013; 13: 304-310.

22. Frank SJ, Johansen MJ, Martirosyan KS et al. A biodistribution and toxicity study of cobalt dichloride $\mathrm{N}$ acetyl cysteine in an implantable MRI marker for prostate cancer treatment. Int J Radiat Oncol Biol Phys 2013; 85: 1024-1030.

23. Lim TY, Stafford RJ, Kudchadker RJ et al. MRI characterization of cobalt dichloride-N-acetyl-cysteine (C4) contrast agent marker for prostate brachytherapy. Phys Med Biol 2014; 59: 2505-2516.

24. Lim TY, Wang J, Kudchadker RJ et al. Effect of pulse sequence parameter selection on signal strength in positive-contrast MRI markers for MRI-based prostate post-implant assessment. Med Phys 2016 [In press].

25. Dhanesar SK, Lim TY, Du W et al. Evaluation of the MIM Symphony treatment planning system for low-dose-rate prostate brachytherapy. J Appl Clin Med Phys 2015; 16: 62-75.

26. Kunos CA, Resnick MI, Kinsella TJ et al. Migration of implanted free radioactive seeds for adenocarcinoma of the prostate using a Mick applicator. Brachytherapy 2004; 3: 71-77.

27. Al-Qaisieh B, Carey B, Ash D et al. The use of linked seeds eliminates lung embolization following permanent seed implantation for prostate cancer. Int J Radiat Oncol Biol Phys 2004; 59: 397-399. 